\title{
ReaR
}

\section{¿Cambia realmente la localización de la membrana cricotiroidea medida en posición neutra con la extensión de la cabeza y cuello? La ecografía nos abre los ojos.}

Artículo original: Dixit A, Ramaswamy K K, Perera S, Sukumar V, Frerk C. Impact of change in head and neck position on ultrasound localisation of the cricothyroid membrane: an observational study. Anaesthesia 2019; 79(1): 29-32. Doi: 10.1111/anae.14445.

Muñoz Ávalos N, Suárez del Arco JA.

\section{HU Puerta de Hierro, Madrid.}

\section{Resumen}

El abordaje quirúrgico de urgencia de la vía aérea ante una situación "no intubable - no ventilable" es un escenario que implica, por parte del anestesiólogo, un adecuado entrenamiento que posibilite una resolución exitosa en el menor tiempo posible. Dada la poca frecuencia de esta práctica, muchos anestesiólogos optan por localizar la membrana cricotiroidea antes de realizar la inducción anestésica con la cabeza y cuello en posición neutra. Ante esto, y, considerando la posición ideal para una cricotiroidotomía al paciente con la cabeza y cuello en extensión, los autores de este artículo tratan de determinar si existe realmente cambio en la localización de dicha membrana con la variación de la cabeza y cuello de posición neutra a extendida así como si se modifica la altura de la misma. Para ello, realizan un estudio observacional con 22 voluntarios sanos a los que un radiólogo, con más de quince años de experiencia, mide con ecografía la distancia entre los bordes superior e inferior de la membrana y realiza una marca en el punto medio de la misma en las dos posiciones antes citadas. Los resultados obtenidos nos llevan a una importante reflexión que nos conduce a un cambio en el paradigma clásico de abordaje de la cricotiroidotomía.

\section{Introducción}

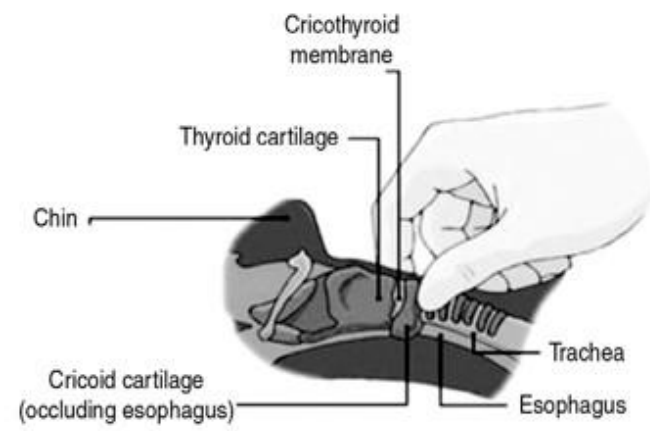

El abordaje quirúrgico de urgencia de la vía aérea ante una situación "no intubable - no ventilable" es un escenario que implica, por parte del anestesiólogo, un adecuado entrenamiento que posibilite una resolución exitosa en el menor tiempo posible. Dada la poca frecuencia de esta práctica, muchos anestesiólogos optan por localizar la membrana cricotiroidea antes de realizar la inducción anestésica con la cabeza y cuello en posición neutra. Ante esto, y, considerando la posición ideal para una cricotiroidotomía al paciente con la cabeza y cuello en extensión, los autores de este artículo tratan de determinar si existe realmente cambio en la localización de dicha membrana con la variación de la cabeza y cuello de posición neutra a extendida así como si se modifica la altura de la misma. Para ello, realizan un estudio observacional con 22 voluntarios sanos a los que un radiólogo, 
con más de quince años de experiencia, mide con ecografía la distancia entre los bordes superior e inferior de la membrana y realiza una marca en el punto medio de la misma en las dos posiciones antes citadas. Los resultados obtenidos nos llevan a una importante reflexión que nos conduce a un cambio en el paradigma clásico de abordaje de la cricotiroidotomía.

El manejo de la vía aérea difícil no deja de ser un reto, a pesar de los importantes avances en vídeo laringoscopia que, en ocasiones, nos lleva a escenarios trágicos en los que el abordaje quirúrgico de la vía aérea se convierte en prioritario.

Afortunadamente, la ecografía va escalando puestos como instrumento de ayuda en este escenario permitiendo resolver problemas que, a menudo, surgen en nuestra práctica diaria, como la localización de la membrana cricotiroidea, objeto de este estudio.

En los algoritmos de las distintas sociedades, como la Sociedad Americana de Anestesiología (ASA), la británica (DAS) y la canadiense (CAFG), se contempla la necesidad de acceso emergente invasivo de la vía aérea en el escenario del paciente "no ventilable - no intubable", por lo que el entrenamiento en esta técnica se convierte en obligado. Muchas veces la incorrecta localización de la membrana cricotiroidea conduce a una técnica fallida (1), por lo que diferentes autores comienzan a destacar el papel de la ecografía, siendo muchos los artículos publicados al respecto, fundamentalmente partiendo de la siguiente premisa comparativa: localizarla mediante palpación versus guía ecográfica. No obstante, no hay ningún estudio publicado entre 1999 y 2017 que establezca diferencia en la localización de la membrana con el cambio de posición de cabeza y cuello así como el efecto que esto conlleva en la altura de la misma. Es por esto que este artículo introduce variables novedosas que tratan de dar explicación a los intentos fallidos de acceso a la membrana cricotiroidea, punto de referencia no solo para la cricotiroidotomía, sino también para la ventilación transtraqueal, la intubación retrógrada así como para la inyección translaríngea de anestésicos locales.

Asimismo, la mayor parte de los artículos publicados en esta línea se basan en estudios sobre cadáveres, punto de dificultad añadido a los autores de este artículo, cuya muestra se compone de voluntarios sanos.

Todo ello hace de este estudio una aportación doblemente innovadora a la bibliografía publicada hasta el momento.

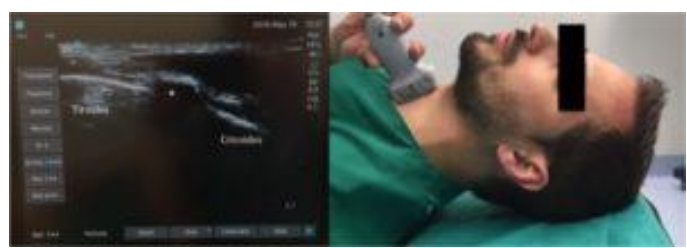

Imagen 1. localización ecográfica de la membrana cricotiroidea. (Fuente: Autores).

\section{Descripción del artículo}

Los autores del artículo que revisamos publicaron un estudio observacional que trataba de responder a dos cuestiones fundamentales cuya finalidad última era mejorar los resultados que, hasta ahora, están asociados a la técnica de cricotiroidotomía. La hipótesis principal de partida pretendía determinar si la marca fijada bajo control ecográfico en el centro de la membrana cricotiroidea en posición neutra varía y se desplaza con la extensión de la cabeza y cuello. La hipótesis secundaria asociada a la previa trataba de valorar si los bordes superior e inferior de la membrana cricotiroidea se modifican con este movimiento.

Para ello, basándose en artículos similares, decidieron tomar como población de estudio a 31 voluntarios sanos, trabajadores del centro hospitalario al que pertenecían los autores, previa 
obtención del consentimiento informado. Los criterios de inclusión se centraron principalmente, en la ausencia de limitación de la movilidad cervical y de neuropatía de miembro superior en sujetos por encima de 18 años. Finalmente, tras la lectura del consentimiento, 7 sujetos decidieron no participar en el estudio y 2 se retiraron por razones logísticas asociadas con el periodo de tiempo de toma de datos, quedando 22 voluntarios como muestra final del estudio. Se recogieron las características antropométricas (peso, talla, circunferencia del cuello) y sexo.

Las mediciones fueron realizadas por un radiólogo de amplia trayectoria profesional con los participantes en el estudio tumbados en una mesa de quirófano, en un primer tiempo, con la cabeza y cuello en posición neutra, marcando la posición central de la membrana y obteniendo medida de los bordes superior e inferior de la misma. En un segundo tiempo, se repitieron las mismas mediciones, pero con la cabeza y cuello en extensión.

El análisis estadístico se llevó a cabo con el test de Wilcoxon mediante el software Sigmaplot 11.0 (Systat, San José, CA, USA). Un valor de $p<0.05$ fue considerado significativo.

Los resultados del estudio mostraron que, con la extensión, la marca central de la membrana se desplazaba y que, en 12 de las 22 medidas iniciales, esa marca quedaba fuera de los límites de la membrana cricotiroidea. Añadido a lo anterior, se obtuvo un aumento del $30 \%$ en el tamaño de la membrana cricotiroidea al cambiar de posición neutra a extendida. No se encontraron diferencias significativas entre el tamaño de la membrana y las características antropométricas de los voluntarios.

\section{Discusión}

Los autores de este artículo parten de una hipótesis original y pragmática, en tanto en cuanto artículos semejantes que han estudiado la localización de la membrana cricotiroidea y su variación de posición con el movimiento han sido realizados sobre una población de estudio diferente (principalmente cadáveres), aspecto que destacan como punto de partida. Así pues, deciden diseñar un estudio observacional, piloto, preliminar en el que toman como muestra final a 22 voluntarios sanos.

Obviamente, en este punto, los propios autores ya reconocen sesgos a la hora de generalizar los resultados de su estudio a la población general, en relación a varios aspectos:

- Son voluntarios que no necesariamente tienen predictores de vía aérea difícil.

- Se toma un tamaño muestral basado en estudios previos similares.

- Se trata de una muestra de participantes sanos en un contexto muy diferente al que habitualmente nos encontramos en quirófano.

Sumado a estas consideraciones se encuentra el hecho, puesto de manifiesto en múltiples estudios (2)(3), de la mayor incidencia de localización incorrecta de la membrana cricotiroidea en mujeres respecto de los hombres, situación que se acentúa a mayor índice de masa corporal, supuesto que los autores tampoco consideran relevante como factor de influencia $y$ posible sesgo en los resultados finalmente obtenidos.

$\mathrm{Si}$ bien es cierto que todas las consideraciones previas son significativas, también lo es que los investigadores de este estudio, conociendo estas limitaciones, pretendan hacer una reflexión y abrir la puerta a nuevas investigaciones que realmente hagan cambiar nuestra pauta de actuación con el 
objetivo de ganar seguridad en el manejo del paciente en un escenario suficientemente dramático como es el paciente "no ventilable-no intubable".

Es evidente que la tecnología nos facilita la práctica diaria y suma seguridad a la misma; en este sentido, la ecografía aplicada a la vía aérea es un gran avance, pero necesita ser aplicado en condiciones óptimas: en este caso, la posición ideal de la cabeza y cuello en la localización y abordaje de la membrana cricotiroidea.

Como ya ha sucedido con los vídeo laringoscopios que han permitido un abordaje de la vía aérea más cómodo, seguro y fiable, la ecografía también demuestra su papel y avanza como técnica auxiliar, pero hemos de tener en cuenta que no todos los centros disponen de ella y que, como se ha visto en la bibliografía publicada, requiere más tiempo en la localización de la membrana que la palpación, por lo que en una situación de emergencia no es la primera medida a considerar. Ello justifica la extensa bibliografía que hay publicada al respecto con comparativas entre diferentes maniobras de palpación para localizar la membrana cricotiroidea (4)(5).

Por todo ello, este artículo trata de justificar el motivo por el cual, ya sea tras palpación de la membrana, o tras su localización mediante ecografía, el acceso incorrecto es el principal motivo de fracaso de la cricotiroidotomía, lo cual, desde nuestro punto de vista, es claramente útil y relevante en nuestra actuación.

\section{Conclusiones}

El manejo de la vía aérea es un pilar fundamental de nuestra actividad diaria $\mathrm{y}$, en muchas ocasiones, nuestro "talón de Aquiles". Tal es así que, de un tiempo a esta parte, ha habido una auténtica revolución tecnológica, con dispositivos cada vez más avanzados que tratan de facilitar y resolver las dificultades que nos encontramos en su abordaje. Aun así, como ponen manifiesto auditorías como el NAP4, hay situaciones extremas que cuentan con una tasa de fallos elevada.

En el tema que nos ocupa, el NAP4 establece que, de entre las técnicas de acceso a la vía aérea, la cricotiroidotomía asocia un bajo porcentaje de éxito. No está del todo claro si es debido a mala colocación, uso incorrecto del dispositivo o uso erróneo del modo ventilatorio tras la inserción, pero la estadística revela la necesidad de revisión de esta técnica. Por ello, insiste, en sus recomendaciones, en que todos los anestesiólogos deben estar entrenados en la cricotiroidotomía de urgencia y en mantener sus habilidades al día para asegurar una técnica óptima.

Es aquí donde este artículo cobra fuerza, poniendo de manifiesto una situación frecuente de fallo de la cricotiroidotomía, que podría aumentar su tasa de éxitos. Localizar la membrana cricotiroidea con la cabeza extendida en vez de en posición neutra, como habitualmente hacemos con carácter anticipatorio antes de la inducción anestésica, y tener presente la posibilidad de emplear cánulas gruesas y tubos de mayor calibre que nos permitan una ventilación más efectiva, según nos pone de manifiesto este artículo que encuentra un espacio más amplio con la extensión de la cabeza del que teníamos conocimiento hasta el momento, cambia la visión, y otorga una nueva perspectiva más optimista de la actuación en estos escenarios.

Por ello creemos que este artículo, aún a pesar de los sesgos citados, podría ser el punto de partida de futuras investigaciones que conduzcan a mayor porcentaje de éxito en situaciones de emergencia de acceso a la vía aérea aumentando así, como fin último, la seguridad de nuestros pacientes. 


\section{Bibliografía}

1. Gadd KJ, Ganguly A, Mauldon EC. The inverted-V shape during transverse laryngeal ultrasonography for cricothyroid membrane localisation. Anaesthesia 2018; 73: 1572-1573. ( $\underline{\text { PubMed }}$ ( $\underline{\text { HTML) }}$

2. Gadd K, Kwok T, Sidhu S, Robertson I. Comparison of two transverse airway ultrasonography techniques for speed and accuracy to localise the cricothyroid membrane in obese female volunteers. British Journal of Anaesthesia 2019; 122 (2): e28-e31. ( PubMed)

3. Alerhand S. Ultrasound for identifying the cricothyroid membrane prior to the anticipated difficult airway. American Journal of Emergency Medicine 2018; 36 (11): 2078-2084. (PubMed)

4. Oh H, Yoon S, Seo M, Oh E, Yoon H, Lee $\mathrm{H}$, et al. Utility of the laryngeal handshake method for identifying the cricothyroid membrane. Acta Anaesthesiologica Scandinavica 2018; 62(9):1223-1228. ( PubMed) (HTML)

5. Drew $\mathrm{T}$, McCaul CL. Laryngeal handshake technique in locating the cricothyroid membrane: a nonrandomised comparative study. British Journal of Anaesthesia 2018; 121 (5): 1173-1178. (PubMed)

Correspondencia al autor

Natalia Muñoz Ávalos

nataliamaval@hotmail.com

FEA Anestesiología, Reanimación y Terapéutica del Dolor.

HU Puerta de Hierro, Madrid.

Aceptado para el blog en septiembre de 2019 